\title{
RNA-seq reveals the downregulated proteins related to photosynthesis in growth-inhibited rice seedlings induced by low-energy $\mathrm{N}^{+}$beam implantation
}

\author{
Q.F. Chen ${ }^{1 *}$, H.Y. Ya ${ }^{1,2 *}$, W.D. Wang ${ }^{1}$ and Z. Jiao ${ }^{1}$ \\ ${ }^{1}$ Henan Provincial Key Laboratory of Ion Beam Bio-Engineering, \\ Zhengzhou University, Zhengzhou, Henan, China \\ ${ }^{2}$ College of Life Sciences Luoyang, Normal University, Luoyang, \\ Henan, China \\ *These authors contributed equally to this study. \\ Corresponding author: H.Y. Ya \\ E-mail: chenqf171@zzu.edu.cn
}

Genet. Mol. Res. 13 (3): 7029-7036 (2014)

Received May 22, 2013

Accepted November 28, 2013

Published March 26, 2014

DOI http://dx.doi.org/10.4238/2014.March.26.9

\begin{abstract}
Transcriptomic research based on RNA sequencing (RNA-seq) technology is innovative and will provide new opportunities and biological perspectives for the use of ion-beam implantation in plants. Using RNA-seq, transcriptomes of whole rice seedlings generated from seeds implanted with a low-energy $\mathrm{N}^{+}$beam were analyzed $96 \mathrm{~h}$ after planting. We identified 544 transcripts that were differentially expressed genes (DEGs) in the non-growth-inhibited sample implanted by an $\mathrm{N}^{+}$beam, including 262 upregulated transcripts and 282 downregulated transcripts. Next, 776 transcripts were identified as DEGs in significantly damaged and growth-inhibited rice seedlings induced by $\mathrm{N}^{+}$beam implantation, including 283 upregulated transcripts and 493 downregulated transcripts. The Gene Ontology (GO) analysis showed that 19 downregulated DEGs in the injured rice seedlings
\end{abstract}


are enriched for the biological process GO term photosynthesis. The Kyoto Encyclopedia of Genes and Genomes pathway analysis also showed that 11 downregulated DEGs related to chlorophyll a/b-binding proteins are statistically significantly enriched in the photosynthesisantenna protein pathway (Pathwayid: map00196). This result suggested that the downregulated and injured photosynthesis system contributed to the growth inhibition of rice seedlings induced by low-energy $\mathrm{N}^{+}$ beam implantation.

Key words: Growth inhibition; Low-energy ion-beam implantation; Transcriptome; RNA-seq; Photosynthesis

\section{INTRODUCTION}

Energetic ion radiation can initiate effects in organisms that absorb the energy and undergo physical, physicochemical, and chemical changes. Some changes include molecular insult, producing visible biological defects. Therefore, radiation biology research is essential to predict such deleterious effects. Studies using low-energy ion-beam biotechnology have been implemented to improve crop growth, microbial resistance, and environmental outcomes that have significant economic and societal benefits. Although low-energy ion-beam plant seed implantation has obvious benefits, such energy can induce mutagenesis, injury, and plant death (Tanaka et al., 2010). The dwarf, strip leaf, growth inhabitation, stimulation growth, abnormal flowers, and abnormal leaf shape variations of plants induced by low-energy radiation can be observed in the first generation (Shikazono et al., 2001; Feng et al., 2007; Ya et al., 2010). Most of these variations were not inherited by the next generation in the crops that were bred by implantation with a low-energy ion beam, which suggested that these variations were caused by abnormal regulation of gene expression. Therefore, gene expression profiles in the plants implanted by a low-energy ion beam are critical to fully understand the biological effects of low-energy ion-beam implantation. However, insufficient research exists to fully describe the mechanisms by which low-energy ion-beam implantation affects plants, especially mechanisms by which low-energy implantation can give rise to many kinds of phenotypic variations in the first generation. Transcriptomics research based on RNA sequencing (RNA-seq) technology will be innovative and will provide new opportunities and biological perspectives for the use of ion-beam implantation in plants (Wang et al., 2009).

Previous research on ion-beam implantation focused on the immediate cause of the mutagenesis (Shikazono et al., 2001; Maekawa et al., 2003; Yamaguchi et al., 2003). Seldom investigated was the molecular mechanism of generational effects of ion-beam irradiation because first-generation biological effects are rarely passed on to subsequent generations. Studying molecular mechanisms behind ion-beam implantation effects can inform studies of mutagenesis mechanisms, specifically those that inhibit plant growth (injury) that is induced by low-energy ion-beam implantation. Most plant mutants that are induced by low-energy ion-beam implantation are derived from first-generation injuries. Using RNA-seq technology to explore gene expression profiles related to inhibited plant growth that is induced by low-energy ion-beam irradiation, we can speculate about plant cellular events based on the metabolism or biological processes that are affected by differential gene expression. 


\section{MATERIAL AND METHODS}

\section{Plant material}

We cultivated Xindao-18 (Oryza sativa L. subsp japonica Kato) rice seeds to mature seedlings and collected the panicle. We removed the glume manually to avoid injury to the rice embryo. Then, complete and similarly sized seeds were selected as experimental material.

\section{Low-energy ion-beam irradiation}

To irradiate seeds with a low-energy ion beam, rice seeds (moisture content: 6.7\%) were vertically fixed on a cork with the embryo facing upward. The cork was then placed on a culture dish $(10 \times 10 \mathrm{~cm})$. Samples were divided into six groups, and five of these six groups were implanted by an $\mathrm{N}^{+}$beam (current intensity of $2 \mathrm{~mA}$, energy of $40 \mathrm{Kev}$ ) with ion fluences of $1 \times 10^{17}, 2 \times 10^{17}, 4 \times 10^{17}, 6 \times 10^{17}$, and $8 \times 10^{17} \mathrm{~N}^{+} / \mathrm{cm}^{2}$. Ion implantation was conducted in a vacuum $\left(10^{-2} \mathrm{MPa}\right)$ target chamber [implanter model: Ion Beam Bioengineering Facility (UIL.0.512, TNV, Russia)]. A seed group, which was not implanted with low energy, served as control $\left(4 \times 10^{17} \mathrm{~N}^{+} / \mathrm{cm}^{2}\right)$. Each group was replicated three times, and each replication contained 150 rice seeds.

\section{Biomaterial cultivation and germination rate, seedling height, and root length measurements}

An aseptic filter paper was placed on the bottom of an aseptic culture dish moistened with aseptic water. Implanted seeds and control seeds were placed on the filter paper, and then the culture dish was placed in a climate box (12-h light/12-h dark cycles) with a constant temperature $\left(28^{\circ} \mathrm{C}\right)$. Every $12 \mathrm{~h}, 10 \mathrm{~mL}$ aseptic water was uniformly dripped on the filter paper of the culture dish to maintain moisture. After culturing for $96 \mathrm{~h}$, we randomly collected 100 seed buds [including bud $(\sim 3 \mathrm{~cm})$ and root] from the sample replicates that were treated with ionbeam irradiation. Buds $(\mathrm{N}=100)$ were stored at $-80^{\circ} \mathrm{C}$ and later sequenced and subjected to total RNA extraction of mixed strains. Remaining buds continued in culture to the seventh day $(168 \mathrm{~h})$. The seed germination rate (germination number/total seeds), seedling height (length from hypocotyl to top of the longest leaf), and root length (length of the longest root: length from hypocotyl to root tip) of these buds were measured.

\section{Preparation of sequencing samples}

Because a 7-day germination should provide ample time for early gene expression, rice buds cultured for $96 \mathrm{~h}$ were selected as material for RNA extraction and RNA-seq. Fifty of the above 100 buds that were cultured for $96 \mathrm{~h}$ and were similar in height from each treated material group were used for the total RNA pool extraction. In experiments, we had one control RNA pool (the first sequencing sample) and five RNA pools from the five groups treated with ion fluences of $1 \times 10^{17}, 2 \times 10^{17}, 4 \times 10^{17}, 6 \times 10^{17}$, and $8 \times 10^{17} \mathrm{~N}^{+} / \mathrm{cm}^{2}$. Based on measured biological effects, $10 \mu \mathrm{g}$ RNA from each of two RNA pools from two ion-beam implanted groups with ion fluences of $1 \times 10^{17}$ and $2 \times 10^{17} \mathrm{~N}^{+} / \mathrm{cm}^{2}$ was mixed to form the second 
sequencing sample (NGI: no significant growth inhibition differences compared to control). Next, $10 \mu \mathrm{g}$ RNA from each of two RNA pools from two ion-beam-implanted groups with ion fluences of $6 \times 10^{17}$ and $8 \times 10^{17} \mathrm{~N}^{+} / \mathrm{cm}^{2}$ were combined to form the third sequencing sample (GI: significant growth inhibition compared to control).

\section{RNA extraction, cDNA library preparation, and sequencing}

Total RNA was extracted from whole seedlings using Trizol reagent (Invitrogen, Carlsbad, CA, USA) and purified with the RNeasy Plant Mini Kit (Qiagen, Valencia, CA, USA). The RNA quality was verified on a Bioanalyzer 2100 (Aligent, Santa Clara, CA, USA); RNA integrity number values were $>8.5$ for all samples. Sequencing libraries were prepared according to manufacturer instructions (Illumina, San Diego, CA, USA). Poly-A-containing mRNA was isolated from the total RNA, subjected to two purification rounds using poly-T oligo-attached magnetic beads, and fragmented using an RNA fragmentation kit. First-strand cDNA was generated using reverse transcriptase and random primers. After second-strand cDNA synthesis and adaptor ligation, 200-bp cDNA fragments were isolated using gel electrophoresis and amplified with 18 cycles of polymerase chain reaction. Products were loaded onto an Illumina HiSeq2000 instrument and subjected to 100 cycles of paired-end ( 2 x $100 \mathrm{bp})$ sequencing. Processing of fluorescent images into sequences, base-calling, and quality value calculations were performed using the Illumina data processing pipeline (version 1.8). The sequence reads were submitted to GenBank GEO database under accession number GSE45908 (http://www.ncbi.nlm.nih.gov/geo/query/acc.cgi?acc=GSE45908).

\section{Mapping of short reads and assessment of differential gene expression}

Raw reads were filtered to obtain high-quality reads by removing low-quality reads. The high-quality reads were mapped onto the Nipponbare reference genome (MSU v7.0) using Tophat v2.0.5. Differential expression was estimated and tested with the package edgeR software (R version: 2.14, edgeRversion: 2.3.52). Gene expression was quantified in terms of reads per kilobase per million mapped reads (RPKM; total exon reads/mapped reads [millions] $\mathrm{x}$ exon length $[\mathrm{kb}]$ ). Transcripts with a false-discovery rate (FDR) $\leq 0.05$ and an estimated absolute $\log 2$ (fold-change) $\geq 1$ were considered to be significantly differentially expressed.

\section{RESULTS}

\section{Descriptive statistics of seed germination rate, seedling height, and root length}

Data revealed no statistically significant differences in the seed germination rate between implanted samples and control despite the seed germination rates of all implanted samples being greater than that of control.

In comparisons of seedling root lengths (Figure 1A), seedling heights of seeds treated with ion fluences of $6 \times 10^{17}$ and $8 \times 10^{17} \mathrm{~N}^{+} / \mathrm{cm}^{2}$ were significantly different from those of controls, but no statistically significant differences in seedling heights were observed with ion fluence treatments of $1 \times 10^{17}, 2 \times 10^{17}$, and $4 \times 10^{17} \mathrm{~N}^{+} / \mathrm{cm}^{2}$ compared to controls. Thus, seedling root growth may be inhibited by ion fluences of $6 \times 10^{17}$ and $8 \times 10^{17} \mathrm{~N}^{+} / \mathrm{cm}^{2}$. 
In comparisons of seedling heights (Figure 1B), a statistically significant difference was observed in samples treated with ion fluences of $4 \times 10^{17}, 6 \times 10^{17}$, and $8 \times 10^{17} \mathrm{~N}^{+} / \mathrm{cm}^{2}$ compared to controls. No statistically significant differences in seedling heights of samples treated with ion fluences of $1 \times 10^{17}$ and $2 \times 10^{17} \mathrm{~N}^{+} / \mathrm{cm}^{2}$ were observed compared to controls. Seedling height appears to be inhibited by ion fluences of $4 \times 10^{17}, 6 \times 10^{17}$, and $8 \times 10^{17} \mathrm{~N}^{+} / \mathrm{cm}^{2}$.

A

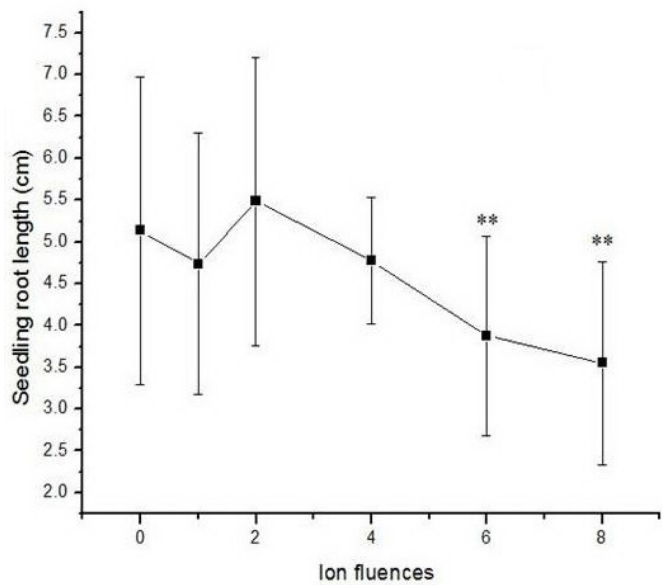

B

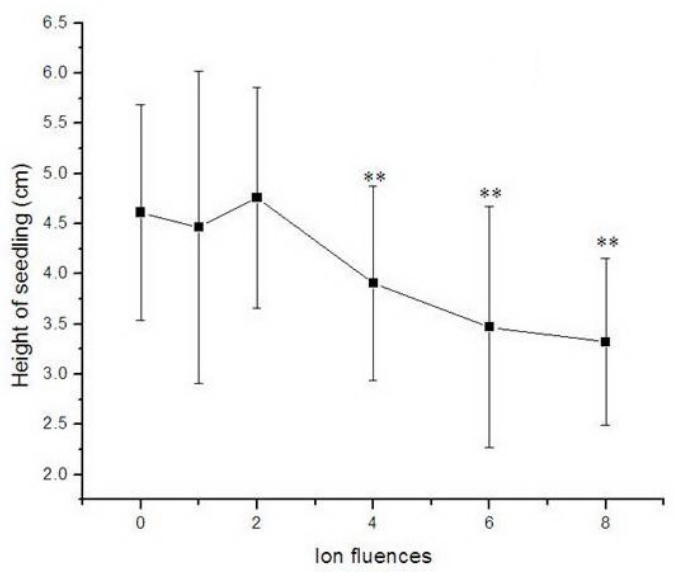

Figure 1. Comparisons of seedling height and root length among different groups. A. Seedling root length. B. Seedling height. $0,1,2,4,6,8$ refer to treatments: blank control, ion fluence of $1 \times 10^{17}, 2 \times 10^{17}, 4 \times 10^{17}, 6 \times 10^{17}$, $8 \times 10^{17} \mathrm{~N}^{+} / \mathrm{cm}^{2}$, respectively. ${ }^{*}$ Significant difference with $\mathrm{P}<0.01$.

Our data led us to hypothesize that the stronger ion fluences of $6 \times 10^{17}$ and $8 \times 10^{17}$ $\mathrm{N}^{+} / \mathrm{cm}^{2}$ could induce biological damage, but ion fluences of $1 \times 10^{17}$ and $2 \times 10^{17} \mathrm{~N}^{+} / \mathrm{cm}^{2}$ did not injure seedlings. According to this result, we prepared three sequencing samples: control, NGI (sample with no significant growth inhibition; mixed RNAs from the samples implanted by ion fluences of $1 \times 10^{17}$ and $2 \times 10^{17} \mathrm{~N}^{+} / \mathrm{cm}^{2}$ ) and GI (sample with significant growth inhibition; mixed RNAs from the samples implanted by ion fluences of $6 \times 10^{17}$ and $8 \times 10^{17} \mathrm{~N}^{+} / \mathrm{cm}^{2}$ ).

\section{Mapping reads to the rice genome}

Paired-end sequencing was performed on the 3 samples using the Illumina HiSeq2000 platform, generating 12,792,715 (control), 12,170,872 (NGI), and 19,177,117 (GI) 2 x 100-bp reads. Raw sequence data are available at the National Center for Biotechnology Information (NCBI) Sequence Read Archive (GSE45908) for further analysis (see Methods). We pooled the short reads and aligned them against the Nipponbare reference genome (MSU V7) using Tophat v2.0.5. Of the reads, 64.4-72.4\% were mapped to exonic regions, $19.1-20.6 \%$ to untranslated regions, $2.36-2.49 \%$ to intronic regions, and $4.95-14.0 \%$ to intergenic regions.

\section{Differentially expressed gene (DEG) identification}

DEGs were derived from comparisons of expressed genes in NGI and control samples 
and from comparisons of expressed genes in GI and control samples. Gene expression was measured as RPKM. The numbers of identified DEGs are listed in Table 1.

Table 1. Differentially expressed genes (DEGs).
\begin{tabular}{lccc} 
\\
\hline Comparisons & DEGs (N) & Upregulated transcripts & Downregulated transcripts \\
\hline NGI $v$ s control & 554 & 262 & 282 \\
GI $v$ control & 776 & 283 & 493 \\
GI $v$ NGI & 726 & 352 & 374 \\
\hline
\end{tabular}

NGI = sample with no-significant growth inhibition; GI = sample with significant growth inhibition .

\section{Functional classification by Gene Ontology (GO)}

AgriGO was used for the functional classification of DEGs, and 307 of the 554 DEGs in NGI vs control were classified into $83 \mathrm{GO}$ molecular function terms, $21 \mathrm{GO}$ cellular component terms, and $120 \mathrm{GO}$ biological process category terms (Du et al., 2010). Only 6 terms in molecular function categories were significant (FDR $<0.05$ ). For GI $v s$ control, 453 of 773 DEGs were classified into $96 \mathrm{GO}$ molecular function terms, 28 cellular component terms, and 130 biological process category terms. Of these, 14 terms in molecular function categories, 14 biological process categories, and 2 cellular component categories were statistically significant (Table 2). In the biological process category, photosynthesis, response, and energy were prominently represented.

\begin{tabular}{|c|c|c|c|c|c|}
\hline GO term & Ontology & Description & Number of DEGs & $P$ value & FDR \\
\hline GO:0009765 & $\mathrm{P}$ & Photosynthesis, light harvesting & 11 & $2.90 \mathrm{E}-15$ & $2.00 \mathrm{E}-12$ \\
\hline GO:0019684 & $\mathrm{P}$ & Photosynthesis, light reaction & 13 & $1.50 \mathrm{E}-12$ & $5.40 \mathrm{E}-10$ \\
\hline GO:0015979 & $\mathrm{P}$ & Photosynthesis & 16 & $7.50 \mathrm{E}-11$ & $1.80 \mathrm{E}-08$ \\
\hline GO:0006979 & $\mathrm{P}$ & Response to oxidative stress & 18 & $4.00 \mathrm{E}-08$ & 7.00E-06 \\
\hline GO:0006091 & $\mathrm{P}$ & Generation of precursor metabolites and energy & 21 & $6.00 \mathrm{E}-07$ & $8.50 \mathrm{E}-05$ \\
\hline GO:0042221 & $\mathrm{P}$ & Response to chemical stimulus & 20 & $2.30 \mathrm{E}-06$ & 0.00027 \\
\hline GO:0010876 & $\mathrm{P}$ & Lipid localization & 11 & $4.50 \mathrm{E}-06$ & 0.00035 \\
\hline GO:0006950 & $\mathrm{P}$ & Response to stress & 38 & $4.40 \mathrm{E}-06$ & 0.00035 \\
\hline GO:0006869 & $\mathrm{P}$ & Lipid transport & 11 & $4.50 \mathrm{E}-06$ & 0.00035 \\
\hline GO:0050896 & $\mathrm{P}$ & Response to stimulus & 40 & $2.20 \mathrm{E}-05$ & 0.0016 \\
\hline GO:0005975 & $\mathrm{P}$ & Carbohydrate metabolic process & 35 & $3.30 \mathrm{E}-05$ & 0.0021 \\
\hline GO:0071669 & $\mathrm{P}$ & Plant-type cell wall organization or biogenesis & 5 & 0.00034 & 0.019 \\
\hline GO:0009664 & $\mathrm{P}$ & Plant-type cell wall organization & 5 & 0.00034 & 0.019 \\
\hline GO:0055114 & $\mathrm{P}$ & Oxidation reduction & 14 & 0.00048 & 0.024 \\
\hline GO:0016684 & $\mathrm{F}$ & Oxidoreductase activity, acting on peroxide as acceptor & 18 & $6.80 \mathrm{E}-08$ & $1.10 \mathrm{E}-05$ \\
\hline GO:0009055 & $\mathrm{F}$ & Electron carrier activity & 44 & $5.40 \mathrm{E}-08$ & $1.10 \mathrm{E}-05$ \\
\hline GO:0004601 & $\mathrm{F}$ & Peroxidase activity & 18 & $6.80 \mathrm{E}-08$ & $1.10 \mathrm{E}-05$ \\
\hline GO:0016209 & $\mathrm{F}$ & Antioxidant activity & 18 & $2.50 \mathrm{E}-07$ & $3.10 \mathrm{E}-05$ \\
\hline GO:0004553 & $\mathrm{F}$ & Hydrolase activity, hydrolyzing O-glycosyl compounds & 26 & $9.00 \mathrm{E}-07$ & $9.00 \mathrm{E}-05$ \\
\hline GO:0016798 & $\mathrm{F}$ & Hydrolase activity, acting on glycosyl bonds & 27 & $1.90 \mathrm{E}-06$ & 0.00016 \\
\hline GO:0020037 & $\mathrm{F}$ & Heme binding & 28 & $3.10 \mathrm{E}-05$ & 0.0022 \\
\hline GO:0005506 & $\mathrm{F}$ & Iron ion binding & 31 & $4.00 \mathrm{E}-05$ & 0.0022 \\
\hline GO:0046906 & $\mathrm{F}$ & Tetrapyrrole binding & 28 & $3.80 \mathrm{E}-05$ & 0.0022 \\
\hline GO:0016491 & $\mathrm{F}$ & Oxidoreductase activity & 63 & 0.00011 & 0.0054 \\
\hline GO:0048037 & $\mathrm{F}$ & Cofactor binding & 23 & 0.00037 & 0.017 \\
\hline GO:0008171 & $\mathrm{F}$ & O-methyltransferase activity & 6 & 0.0005 & 0.021 \\
\hline GO:0016829 & $\mathrm{F}$ & Lyase activity & 14 & 0.0012 & 0.045 \\
\hline GO:0005507 & $\mathrm{F}$ & Copper ion binding & 9 & 0.0013 & 0.046 \\
\hline GO:0005576 & $\mathrm{C}$ & Extracellular region & 20 & $2.80 \mathrm{E}-07$ & $3.10 \mathrm{E}-05$ \\
\hline GO:0048046 & $\mathrm{C}$ & Apoplast & 10 & $1.10 \mathrm{E}-05$ & 0.00062 \\
\hline
\end{tabular}

$\mathrm{FDR}=$ false-discovery rate $\mathrm{F}=$ molecular function category; $\mathrm{C}=$ cellular component $\mathrm{P}=$ biological process category. 


\section{Kyoto Encyclopedia of Genes and Genomes (KEGG) pathway analysis}

To identify metabolic pathways responsive to ion-beam implantation adaptation, pathway-based analysis was performed using the KEGG pathway database. No significant pathways of DEGs were evident in GI $v s$ control or in NGI $v s$ control, except for the photosynthesis-antenna protein pathway (Pathwayid: map00196) in GI. No significantly enriched pathways were found to be involved in DEGs when NGI was compared to control. The 11 downregulated DEGs (chlorophyll a/b-binding proteins) involved in the photosynthesis pathway were expressed lower in the GI sample than in the control sample (Table 3).

\begin{tabular}{lcc}
\multicolumn{2}{c}{ Table 3. Downregulated differentially expressed genes involved in the photosynthesis pathway. } \\
\hline Gene_id & $\log 2$ (fold-change) GI $v s$ control & $\log 2$ (fold-change) GI $v s$ NGI \\
\hline LOC_Os01g41710 & -5.12 & -5.31987 \\
LOC_Os02g10390 & -4.46 & -5.13882 \\
LOC_Os03g39610 & -6.25 & -6.60422 \\
LOC_Os04g38410 & -2.13 & -2.7851 \\
LOC_Os06g21590 & -2.09 & -2.73821 \\
LOC_Os07g37240 & -1.52 & -1.91876 \\
LOC_Os07g37550 & -2.85 & -3.25119 \\
LOC_Os07g38960 & -1.63 & -2.12569 \\
LOC_Os08g33820 & -4.38 & -4.88249 \\
LOC_Os09g17740 & -5.94 & -6.27338 \\
\hline
\end{tabular}

GI = sample with significant growth inhibition; NGI = sample with no-significant growth inhibition.

\section{DISCUSSION}

Light-harvesting chlorophyll a/b-binding protein (LHCP) is one of the most abundant chloroplast proteins found in plants. LHCP's chief function is to collect and transfer light energy to photosynthetic reaction centers (Xia et al., 2012). Many homologous genes encoding LHCPs from various plant species belong to 1 of the 10 gene family members (Teramoto et al., 2001). Under changing light conditions, the reversible phosphorylation of LHCP of photosystem II represents a system for balancing excitation energy between photosystems I and II (Liu and Shen, 2004). The 11 down-regulated DEGs involved in a photosynthesis pathway were less expressed in NGI and GI samples than in the control sample. Such diminished expression reduces light harvesting and synthesis of plant energy. In agreement with this concept, seedling root length and height were diminished in GI, suggesting that the growth of samples treated with ion fluences of $6 \times 10^{17}$ and $8 \times 10^{17} \mathrm{~N}^{+} / \mathrm{cm}^{2}$ was inhibited by downregulated gene expression related to LHCP.

Additionally, the construction of the gene expression network using a web service (http://www.ricearray.org/coexpression/coexpression.shtml) shows that 7 of these 11 DEGs related to LHCPs are regulated by two $\mathrm{C} 2 \mathrm{C} 2-\mathrm{CO}$-like transcription factors (Table 4), which suggests the unified regulation of these genes.

Table 4. Construction of the gene expression network about differentially expressed genes (partially shown).

\begin{tabular}{lccc}
\hline Transcription factor gene & Transcription factor class & Target gene & PCC value \\
\hline LOC_Os02g05470 & C2C2-CO-like & LOC_Os07g37550 & 0.7827 \\
LOC_Os02g05470 & C2C2-CO-like & LOC_Os02g10390 & 0.8124 \\
LOC_Os06g15330 & C2C2-CO-like & LOC_Os07g38960 & 0.7738 \\
LOC_Os06g15330 & C2C2-CO-like & LOC_Os03g39610 & 0.7816 \\
LOC_Os06g15330 & C2C2-CO-like & LOC_Os04g38410 & 0.7881 \\
LOC_Os06g15330 & C2C2-CO-like & LOC_Os08g33820 & 0.8044 \\
LOC_Os06g15330 & C2C2-CO-like & LOC_Os06g21590 & 0.8584 \\
LOC_Os06g24070 & GARP-G2-like & LOC_Os07g37240 & 0.8454 \\
\hline
\end{tabular}

$\mathrm{PCC}=$ co-expression Pearson correlation coefficent (cutoff beyond 0.75 ). 


\section{ACKNOWLEDGMENTS}

Research supported by the Key Technology Projects of Henan Province (\#10210110108, \#122300410045), the program-funded Project for Young Teachers in Universities of Henan Province (\#2010ggjs-168), and the National Natural Science Fund of China (\#30800204 and \#U1204307).

\section{REFERENCES}

Du Z, Zhou X, Ling Y, Zhang Z, et al. (2010). agriGO: a GO analysis toolkit for the agricultural community. Nucleic Acids Res. 38: W64-W70.

Feng HY, Liu XL, Yuan H, Kong MG, et al. (2007). Utilizing low-energy ion beams to study living organisms. Surf. Coat. Technol. 201: 8034-8038.

Liu XD and Shen YG (2004). NaCl-induced phosphorylation of light harvesting chlorophyll a/b proteins in thylakoid membranes from the halotolerant green alga, Dunaliella salina. FEBS Lett. 569: 337-340.

Maekawa M, Hase Y, Shikazono N and Tanaka A (2003). Induction of somatic instability in stable yellow leaf mutant of rice by ion beam irradiation. Nucl. Instrum. Methods 206: 579-585.

Shikazono N, Tanaka A, Watanabe H and Tano S (2001). Rearrangements of the DNA in carbon ion-induced mutants of Arabidopsis thaliana. Genetics 157: 379-387.

Tanaka A, Shikazono N and Hase Y (2010). Studies on biological effects of ion beams on lethality, molecular nature of mutation, mutation rate, and spectrum of mutation phenotype for mutation breeding in higher plants. J. Radiat. Res. 51: 223-233.

Teramoto H, Ono T and Minagawa J (2001). Identification of $L h c b$ gene family encoding the light-harvesting chlorophyll$a / b$ proteins of photosystem II in Chlamydomonas reinhardtii. Plant Cell Physiol. 42: 849-856.

Wang Z, Gerstein M and Snyder M (2009). RNA-Seq: a revolutionary tool for transcriptomics. Nat. Rev. Genet. 10: $57-63$.

Xia YS, Ning ZX, Bai GH, Li RH, et al. (2012). Allelic variations of a light harvesting chlorophyll A/B-binding protein gene (Lhcb1) associated with agronomic traits in barley. PLoS One 7: e37573.

Ya HY, Wang WD, Chen WG, Qin GY, et al. (2010). Retrotransposon Ttol in tobacco was activated by the implantation of low-energy $\mathrm{N}^{+}$ion beam. Life Sci. J. 7: 141-147.

Yamaguchi H, Nagatomi S, Morishita T, Degi K, et al. (2003). Mutation induced with ion beam irradiation in rose. Nucl. Instrum. Methods B 206: 561-564. 